Auch und gerade angesichts schockierender Gewaltverbrechen sind sachliche Beurteilungen gefragt. Tatsache ist, dass die vertrauensvolle Arzt-Patient-Beziehung eine unverzichtbare Basis für die wirksame Behandlung und korrekte Einschätzung von Straftätern ist. Das Arztgeheimnis trägt damit entscheidend zur öffentlichen Sicherheit bei. Die FMH unterstützt darum den Walliser Ärzteverband, der einer letztlich gefährlichen Meldepflicht entgegentritt.

Dr. med. Jürg Schlup, Präsident der FMH

\title{
Aufweichung des Arztgeheimnisses gefährdet öffentliche Sicherheit
}

Die Morde an Adeline und Marie in den Kantonen Waadt und Genf durch Wiederholungstäter im Jahre 2013 lösen berechtigter Weise starke Emotionen aus. Umso wichtiger ist es, dass keine emotionsbasierten Gesetzesänderungen umgesetzt werden, die dem politischen Druck entspringen, aber nicht zielführend sind. Wie in den Kantonen Genf und Waadt werden auch den Walliser Parlamentariern Anpassungen des Einführungsgesetzes zum Schweizerischen Strafgesetzbuch (EGStGB) vorgeschlagen, die für behandelnde Ärzte eine Meldepflicht von als gefährlich eingestuften Straftätern beinhalten. Diese verlangt, dass die Gesundheitsfachperson «die zuständigen Verwaltungs- und Justizbehörden über alle wichtigen Tatsachen zu informieren hat, welche einen Einfluss auf die laufenden Massnahmen (...) oder im Allgemeinen auf die Beurteilung der Gefährlichkeit der betroffenen Person haben können». Dieser Vorschlag ist unverhältnismässig und kontraproduktiv. Er führt zur Aufweichung des Arztgeheimnisses und verunmöglicht damit den Aufbau eines Vertrauensverhältnisses zum betroffenen Häftling. Deswegen erhält der behandelnde Arzt keine relevanten Informationen mehr von diesem, sodass eine angemessene Behandlung nicht möglich ist. Durch den induzierten Informationsverlust kann der Arzt auch eine potentielle Gefahrensituation verkennen, die er heute, basierend auf dem bestehenden Melderecht, hätte weiterleiten können. Stattdessen wird er in die Rolle eines Experten für die Beurteilung der sogenannten «Gefährlichkeit»

\section{Meldepflicht bei Straftätern weicht das Arztgeheimnis auf und gefährdet die Sicherheit der Bevölkerung.}

des Häftlings und für die Adäquanz der laufenden strafgesetzlichen Massnahmen gedrängt. Dieses Doppelmandat ist schon aus rechtlicher Sicht nicht statthaft. Aus fachlicher Sicht ist dieses widersprüchliche Mandat schlicht nicht umsetzbar! Dank Interventionen der Ärzteschaft anlässlich früherer Anhörungen haben die Politiker in den Kantonen Genf und Waadt den Stellenwert der Problematik erkannt und ver- langen die korrekte Integration der genannten Kritikpunkte in Bezug auf das Arztgeheimnis im Gesetzesentwurf.

Anlässlich der Anhörung im Kanton Wallis durch die zuständige parlamentarische Kommission hat der Walliser Ärzteverband dargelegt, weshalb der Vorentwurf durch die Ärzteschaft vehement abgelehnt wird. Wir wurden dabei durch Frau Monique Gauthey, Fachärztin und Mitglied des FMHZentralvorstandes, sowie durch Hrn. Prof. Urs Saxer, Jurist der

\section{Die Optimierung des Informationsaus- tausches zwischen Ärzten und Behör- den benötigt keine Gesetzesänderung.}

KKA, unterstützt. Gemeinsam konnten wir einerseits darlegen, welche zentrale Bedeutung dem Arztgeheimnis auch bei Häftlingen zukommt und andererseits, dass eine Verbesserung der Informationsprozesse bereits durch eine einfache Formalisierung bestehender Abläufe erreichbar und hierfür keine Gesetzesänderung notwendig wäre. Wir hoffen, dass diese Anmerkungen der Ärzteschaft nun auch im Wallis entsprechend in der weiteren Gesetzesanpassung berücksichtigt werden.

Der Walliser Ärzteverband wird sich weiterhin engagieren, um eine optimale Lösung für alle Beteiligten zu finden, die das Arztgeheimnis respektiert und eine korrekte Behandlung von als gefährlich eingestuften Straftätern auch im Wallis ermöglicht. Auch in Hinblick darauf, dass die meisten dieser Häftlinge eines Tages entlassen werden, ist eine adäquate medizinische Behandlung nicht verhandelbar. Es ist unerlässlich, dass die fundierten Sicherheitsbedenken der Ärzteschaft im Bereich des Arztgeheimnisses korrekt in den aktuell laufenden Vernehmlassungen berücksichtigt werden und das Arztgeheimnis nicht zum Spielfeld politischer Interessen verkommt.

Dr. med. Monique Lehky Hagen, Präsidentin des Walliser Ärzteverbandes 